\title{
Optimising psychotropic medication use
}

Rory Sheehan

\section{Abstract}

Purpose

This commentary accompanies Clare et al's study investigating psychotropic drug prescribing for adults with intellectual disability who were referred to specialist community learning disability teams in the east of England. The aim is to explore some of the background to psychotropic drug prescribing for people with intellectual disability, review important contextual factors involved that influence prescribing decisions, and consider how we might make the best use of psychotropic drugs in this group.

\section{Design/methodology/approach}

Narrative summary and opinion, supported by reference to recent research literature.

\section{Findings}

Psychotropic drug use for people with intellectual disability is complex, not least because of the lack of research evidence that exists on the topic. Psychotropic drugs can be an important part of treatment for people with mental illness but further research is needed to support prescribing for challenging behaviour. Medication optimisation is a framework within which individual preferences and values are considered alongside the evidence-base and clinical judgement in order to inform safe, effective, and collaborative management decisions.

\section{Practical implications}

Prescribing decisions should be individualised and reviewed regularly, incorporating evidence from patients and carers. Improving the use of psychotropic medication requires concerted action, adequate social support, and the provision of alternative, non-pharmacological interventions that are acceptable and effective. 
This paper reviews some of the current concerns about the use of psychotropic drugs and opens up new avenues of discussion.

\section{Keywords}

Intellectual disability; mental illness; challenging behaviour; psychotropic medication; medication optimisation; interventions

$\underline{\text { Article classification }}$

Commentary / viewpoint

\section{Conflicts of interest}

The author reports no conflicts of interest. 


\section{Optimising psychotropic medication use}

The prescribing of psychotropic medication to people with intellectual disability continues to attract interest and be an ongoing source of controversy. The paper by Isabel Clare and colleagues in this edition of the journal is a relatively small-scale investigation of psychotropic medication prescribing in a sample of adults referred to multi-disciplinary intellectual disability services in the east of England. The paper adds evidence from community secondary care to recent research on psychotropic prescribing which has drawn on large electronic primary care databases (Sheehan et al., 2015). The high rate of psychotropic prescribing found by Clare et al. is not a surprise, given what we already know on the subject and the fact that individuals referred to secondary care services will often have complex mental and behavioural presentations. Similarly, the relatively large proportion of the sample who were prescribed psychotropic drugs in the absence of an underlying diagnosis of mental illness is consistent with previous data and may suggest off-license use of medication, in some cases (Paton et al., 2016).

Yet again, this study provokes questions about the place of psychotropic medications in the treatment of adults with intellectual disability. Whereas few would disagree that psychotropic medication is indicated in the management of diagnosed mental disorder there is, in fact, relatively little evidence for the efficacy and safety of these drugs specifically in people with intellectual disability. Knowledge of the effects of psychotropic drugs is extrapolated from evidence of studies of their use in the non-intellectually disabled population, which may have limited external validity. Indeed, there are reasons to believe that the pharmacodynamics of psychotropic drugs are different in people with intellectual disability, possibly owing to interaction with pre-existing cognitive deficits and co-morbid conditions. For example, we recently showed that people with intellectual disability are more susceptible to centrallymediated movement side-effects of antipsychotic drugs, such as akathisia and Parkinsonism (Sheehan et al., 2017). In our study, neuroleptic malignant syndrome, a rare but potentiallyfatal side-effect of antipsychotic drugs was also much more common in people with intellectual disability. Risk factors, the presentation of mental illness and its natural history 
may all be different in people with intellectual disability and not only make accurate diagnosis more difficult but also potentially influence treatment response.

People with intellectual disability are under-represented in medical research and are routinely excluded from clinical studies, either explicitly in study protocols, or indirectly by virtue of trial processes and procedures that disadvantage those with cognitive deficits. Researchers might be deterred by assumptions about the ability of people with intellectual disability to provide informed consent and perceived obstacles in gaining ethical approval for recruitment of participants who lack capacity (Goldsmith and Skirton, 2015). Even studies designed for participants with intellectual disability can face problems recruiting adequate numbers, as clinicians might be reluctant to nominate those who are potentially eligible.

Whilst the use of psychotropic medication for mental illness in people with intellectual disability is generally not disputed, the use of medication for challenging behaviour is more contentious. Challenging behaviour does not have an obvious equivalent in the nonintellectually disabled population and there is little evidence that can be transferred from other groups. The antipsychotic drugs risperidone and aripiprazole show some benefit in the short-term management of challenging behaviour in children (McQuire et al., 2015) but evidence for their use for challenging behaviour in adults with intellectual disability is inconclusive, at best (Deb, 2014). Psychotropic prescribing is not without risks; as mentioned above, adverse side-effects are common and may negatively impact wellbeing (Koch et al., 2015). On this basis, national and international best practice consensus guidelines for the management of challenging behaviour favour psychosocial interventions (NICE, 2015a). Guidelines recognise only a limited place for antipsychotic drugs in the management of challenging behaviour in the absence of a diagnosis of mental illness where, for example, the risk of harm is severe and alternative interventions have failed. Causes of challenging behaviour are multiple and varied, and most often there will not be a single explanation. Psychotropic medication should be used alongside psychosocial interventions with the focus remaining on determining and addressing the cause of the behaviour, rather than merely suppressing it by sedating the individual. 
A small number of cases of challenging behaviour may have biological underpinnings which are legitimate targets for pharmacological manipulation. The opiate hypothesis of self-injury holds that chronically high circulating levels of endogenous opiates or opiate receptor dysregulation reduces sensitivity to pain and the self-injury then becomes a form of sensory stimulation. Self-injury may then be reinforced by the pleasurable, euphoric-like effects induced by the release of endogenous opiates (Sandman and Hetrick, 1995). The opiate hypothesis thus neatly indicates rational management with opiate receptor blockers, such as naltrexone, to attenuate self-injury. However, formal research evidence for the benefit of opiate antagonists used in this context is relatively weak (Rana et al., 2013) suggesting that other factors must also be important.

Behavioural phenotypes, in which genetic or chromosomal conditions are associated with a characteristic pattern of behaviour and neurodevelopmental profile, also seem to suggest an important role of individual constitutional factors in the development and expression of challenging behaviour. However, even where the genetic and molecular basis of the disorder is well-characterised, the pathway to challenging behaviour is often tangential. For example, although the genetic basis of Lesch-Nyhan syndrome and the subsequent metabolic abnormality have been known for several decades, this has not translated to effective treatments for the developmental and behavioural aspects of the disorder and the severe self-injury that characterises this syndrome has been shown to be mediated by environmental factors (Hall et al., 2001).

Aggression is the most prevalent type of challenging behaviour in people with intellectual disability and is a common reason for referral to clinical services (Emerson et al., 2001). The neurobiological underpinnings of aggression have been studied in detail; aberrant conduction in central dopaminergic, serotonergic, and GABA pathways have all been implicated (Willner, 2015). However the neurobiological system is complex and interdependent and targeted pharmacological treatments for aggression have largely been unsuccessful.

Any biological explanations for challenging behaviour are clearly, therefore, only one part of a complex picture. They may contribute to the formulation of certain cases of challenging behaviour but do not negate the social dimensions of behavioural disturbance. Challenging 
behaviour as a concept necessarily involves an interaction with the environment and management strategies that focus only on the individual cannot ever be wholly successful. Effective intervention depends on the implementation of broader strategies that complement one another and consider psychological, social and environmental determinants, as well as potential biological and medical causes.

Translating new scientific advances to everyday care will take time and we still need much more research before the promise of targeted drug therapy may be realised for many people with intellectual disability. More work is needed to explore the topographies of challenging behaviour and subgroups of people who may benefit from psychotropic medication. In the meantime, we must ensure that psychotropics are used pragmatically, whether for mental illness or challenging behaviour. Medicines optimisation is an approach to prescribing that aims to maximise benefit and minimise harm of medication, with a focus on patient experience and outcome (NICE, 2015b). Embedded within this is a central role for the values and preferences of patients (and others who may support or advocate for them) which are considered alongside the scientific evidence base and clinical experience as part of a process of shared decision making. Thus, the concept extends beyond the practical issues of prescribing, such as those of drug administration and potential interactions, that must also be considered (Trollor et al., 2016).

Given the relative lack of evidence, Santosh and Baird suggest treating each case of prescribing for people with intellectual disability as a single case study (Santosh and Baird, 1999). Determining the risks and benefits of medication is contingent on access to good quality data. Positive effects of medication on symptoms or functioning should be recorded and plotted against unwanted or adverse effects, preferably using validated scales (Ramerman et al., 2017). People with intellectual disability often have reduced health literacy and are less likely to recognise or report adverse events and a combination of collateral information, behavioural observations, and clinical assessment are essential in judging the overall impact of a medication. Whilst the paper by Clare and colleagues did not find that polypharmacy or prescription of high doses of antipsychotics were common, other research has shown this to be the case (Bowring et al., 2017) and psychotropic medication is frequently used over extended periods of time (McGillivray and McCabe, 2006). Clinicians and carers 
need to be empowered and prepared to take a critical stance with respect to long-term use of medication. Physical health monitoring, necessary to reduce the risks of iatrogenic harm, is inadequate in people with intellectual disability who are prescribed psychotropic medication (Paton et al., 2016) and requires close collaboration between primary and secondary care.

To improve, and potentially reduce, psychotropic prescribing for challenging behaviour we must recognise the non-clinical influences and competing demands that can impact prescribing decisions. Various upstream factors, such as carer attributions for a behaviour, can heavily influence staff response (Hastings and Remington, 1994). Prescribing does not occur in isolation from other management decisions; psychiatrists express a strong preference for non-medication based intervention as first line management in challenging behaviour in the absence of mental illness (Unwin and Deb, 2008) but may find themselves with few other options where alternative psychosocial strategies are not readily available. Provision of adequate care and support has been highlighted as a factor likely to increase the chances that physicians will attempt to reduce or discontinue antipsychotic drugs used for behaviour (Kuijper and Hoekstra, 2017). Even when medications used for behaviour are reduced, the success or otherwise of withdrawal depends largely on setting rather than individual factors, such as availability of trained support staff (Ahmed et al., 2000).

\section{Conclusions}

Psychotropic medication is an important part of treatment for many people with intellectual disability who have co-morbid mental illness, and might be helpful in select cases of challenging behaviour. As the evidence base for use of psychotropic drugs in people with intellectual disability is limited, prescribing decisions must be made on a case-by-case basis, in collaboration with the person with intellectual disability and those whom they trust to support them. Owing to the likely increased rate of side-effects in this group and the potential for atypical and idiosyncratic reactions, medication should be reviewed regularly with reference to target symptoms and valid sources of data. 
There is now reasonably good evidence that suggests over-use of psychotropic medication in people with intellectual disability. Improving prescribing requires a nuanced approach that recognises the relationships between psychotropic drug use and the wider health and social care environment. It is important that we do not disadvantage people who may stand to benefit from psychotropic drugs. Applying a framework of medication optimisation could promote shared decision making and ensure the best use of psychotropic medication as part of holistic $C_{2}$ person-centred treatment.

\section{References}

Ahmed, Z., Fraser, W., Kerr, M. P., Kiernan, C., Emerson, E., Robertson, J., Felce, D., Allen, D., Baxter, $\mathrm{H}$. and Thomas, J. (2000), "Reducing antipsychotic medication in people with a learning disability", The British Journal of Psychiatry, Vol. 176, No. 1, pp. 42-46.

Bowring, D., Totsika, V., Hastings, R., Toogood, S. and Mcmahon, M. (2017), "Prevalence of psychotropic medication use and association with challenging behaviour in adults with an intellectual disability. A total population study", Journal of Intellectual Disability Research, Vol. 61, No. 6, pp. 604-17.

Deb, S. (2014), "Psychopharmacology", in Tsakanikos, E. and McCarthy, J. (Eds.) Handbook of psychopathology in intellectual disability: research, practice, and policy. Springer: New York.

Emerson, E., Kiernan, C., Alborz, A., Reeves, D., Mason, H., Swarbrick, R., Mason, L. and Hatton, C. (2001), "The prevalence of challenging behaviors: A total population study", Research in Developmental Disabilities, Vol. 22, No. 1, pp. 77-93.

Goldsmith, L. and Skirton, H. (2015), "Research involving people with a learning disabilitymethodological challenges and ethical considerations", Journal of Research in Nursing, Vol. 20, No. 6, pp. 435-46.

Hall, S., Oliver, C. and Murphy, G. (2001), "Self-injurious behaviour in young children with Lesch-Nyhan syndrome", Developmental medicine and child neurology, Vol. 43, No. 11, pp. 745-49.

Hastings, R.P. and Remington, B. (1994), "Staff behaviour and its implications for people with learning disabilities and challenging behaviours", British Journal of Clinical Psychology, Vol. 33, No. 4, pp. 423-38.

Koch, A. D., Vogel, A., Becker, T., Salize, H.-J., Voss, E., Werner, A., Arnold, K. and Schützwohl, M. (2015), "Proxy and self-reported Quality of Life in adults with intellectual disabilities: Impact of psychiatric symptoms, problem behaviour, psychotropic medication and unmet needs", Research in Developmental Disabilities, Vol. 45, pp. 136-46.

Kuijper, G. and Hoekstra, P. (2017), "Physicians' reasons not to discontinue long-term used off-label antipsychotic drugs in people with intellectual disability", Journal of Intellectual Disability Research, e-pub ahead of print.

Mcgillivray, J. A. and Mccabe, M. P. (2006), "Emerging trends in the use of drugs to manage the challenging behaviour of people with intellectual disability", Journal of Applied Research in Intellectual Disabilities, Vol. 19, No. 2, pp. 163-72.

Mcquire, C., Hassiotis, A., Harrison, B. and Pilling, S. (2015), "Pharmacological interventions for challenging behaviour in children with intellectual disabilities: a systematic review and metaanalysis", BMC psychiatry, Vol. 15, 303. 
NICE (2015a), "Challenging behaviour and learning disabilities: prevention and interventions for people with learning disabilities whose behaviour challenges [NG11]", National Institute for Health and Care Excellence, London.

NICE (2015b), "Medicines optimisation: the safe and effective use of medicines to enable the best possible outcomes. NICE Guideline [NG5]", National Institute for Health and Care Excellence, London.

Paton, C., Bhatti, S., Purandare, K., Roy, A. and Barnes, T. (2016), "Quality of prescribing of antipsychotic medication for people with intellectual disability under the care of UK mental health services: a cross-sectional audit of clinical practice", BMJ Open, 6, e013116.

Ramerman, L., Ramerman, L., De Kuijper, G., De Kuijper, G., Hoekstra, P. J. and Hoekstra, P. J. (2017), "Adherence of clinicians to guidelines for the prescription of antipsychotic drugs to people with intellectual disabilities", Advances in Mental Health and Intellectual Disabilities, Vol. 11, No. 3, pp. 110-25.

Rana, F., Gormez, A. and Varghese, S. (2013), "Pharmacological interventions for self-injurious behaviour in adults with intellectual disabilities", Cochrane Database of Systematic Reviews No. 4, CD009084.

Sandman, C. A. and Hetrick, W. P. (1995), "Opiate mechanisms in self-injury", Developmental Disabilities Research Reviews, Vol. 1, No. 2, pp. 130-6.

Santosh, P. J. and Baird, G. (1999), "Psychopharmacotherapy in children and adults with intellectual disability", The Lancet, Vol. 354, No. 9174, pp. 233-42.

Sheehan, R., Hassiotis, A., Walters, K., Osborn, D., Strydom, A. and Horsfall, L. (2015), "Mental illness, challenging behaviour, and psychotropic drug prescribing in people with intellectual disability: UK population based cohort study", BMJ, Vol. 351, h4326.

Sheehan, R., Horsfall, L., Strydom, A., Osborn, D., Walters, K. and Hassiotis, A. (2017), "Movement sideeffects of antipsychotic drugs in people with and without intellectual disability: UK populationbased cohort study", BMJ Open, in press.

Trollor, J. N., Salomon, C. \& Franklin, C. 2016. Prescribing psychotropic drugs to adults with an intellectual disability. Australian Prescriber, Vol. 39, No. 4, pp. 126-30.

Unwin, G. L. and Deb, S. (2008), "Use of medication for the management of behavior problems among adults with intellectual disabilities: A clinicians' consensus survey" American Journal on Mental Retardation, Vol. 113, No. 1, pp. 19-31.

Willner, P. (2015). The neurobiology of aggression: implications for the pharmacotherapy of aggressive challenging behaviour by people with intellectual disabilities. Journal of Intellectual Disability Research, Vol. 59, No. 1, pp. 82-92. 